\title{
Perspectiva bayesiana na seleção de genótipos de feijão-caupi em ensaios de valor de cultivo e uso
}

\author{
Paulo Eduardo Teodoro(1), Moysés Nascimento(2), Francisco Eduardo Torres ${ }^{(1)}$, \\ Laís Mayara Azevedo Barroso ${ }^{(2)}$ e Edvaldo Sagrilo(3)
}

\begin{abstract}
(1)Universidade Estadual de Mato Grosso do Sul, Unidade de Aquidauana, Rodovia Aquidauana, Km 12, Zona Rural, CEP 79200-000 Aquidauna, MS, Brasil. E-mail: eduteodoro@hotmail.com, feduardo@uems.br ${ }^{(2)}$ Universidade Federal de Viçosa, Departamento de Estatística, Avenida

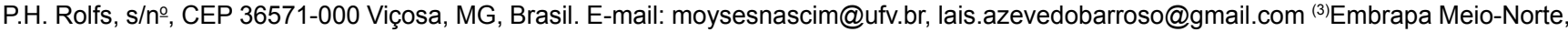
Avenida Duque de Caxias, no 5.650, Buenos Aires, CEP 64006-220 Teresina, PI, Brasil. E-mail: edvaldo.sagrilo@embrapa.br
\end{abstract}

Resumo - O objetivo deste trabalho foi selecionar, sob a perspectiva bayesiana, genótipos de feijão-caupi (Vigna unguiculata) que reúnam alta adaptabilidade e estabilidade fenotípicas, no Estado do Mato Grosso do Sul. Foram utilizados dados de quatro experimentos, conduzidos em delineamento de blocos ao acaso, em que a produtividade de grãos de 20 genótipos de feijão-caupi semiprostrado foi avaliada. Para representar as distribuições a priori pouco informativas, utilizaram-se distribuições de probabilidade com grande variância; e, para representar distribuições a priori informativas, adotou-se o conceito de metanálise, com uso de informações de trabalhos anteriores. A comparação entre as distribuições a priori foi realizada por meio do fator de Bayes. A abordagem bayesiana proporciona maior acurácia na seleção de genótipos de feijão-caupi semiprostrado, com elevadas adaptabilidade e estabilidade fenotípicas avaliadas por meio da metodologia de Eberhart \& Russell. Com base nas prioris informativas, os genótipos MNC99-507G-4, TE97-309G-24, MNC99-542F-7 e BR 17-Gurguéia são classificados como de alta adaptabilidade a ambientes favoráveis. Já os genótipos TE96-290-12G, MNC99-510F-16, MNC99-508G-1, MNC99-541F-21, MNC99-542F-5 e MNC99-547F-2 apresentam alta adaptabilidade a ambientes desfavoráveis.

Termos para indexação: Vigna unguiculata, fator de Bayes, interação genótipo x ambiente, metanálise, priori informativa.

\section{Bayesian perspective in the selection of cowpea genotypes in trials of value for cultivation and use}

\begin{abstract}
The objective of this work was to select, under the Bayesian perspective, cowpea (Vigna unguiculata) genotypes that meet high phenotypic adaptability and stability, in the state of Mato Grosso do Sul, Brazil. Data from four experiments, conducted in a randomized complete block design, were used, in which grain yield of 20 semiprostrate cowpea genotypes was evaluated. To represent non-informative prior distributions, probability distributions with high variance were used; and, to represent informative prior distributions, a metanalysis concept was adopted using information from previous studies. The comparison between the prior distributions was done using the Bayes factor. The Bayesian approach provides greater accuracy in the selection of semiprostrate cowpea genotypes, with high phenotypic adaptability and stability assessed by the Eberhart \& Russell methodology. Based on the informative priors, the MNC99-507G-4, TE97-309G-24, MNC99-542F-7, and BR 17-Gurguéia genotypes are classified as with high adaptability to favorable environments. The TE96-290-12G, MNC99-510F-16, MNC99-508G-1, MNC99-541F-21, MNC99-542F-5, and MNC99-547F-2 genotypes have high adaptability to unfavorable environments.
\end{abstract}

Index terms: Vigna unguiculata, Bayes factor, genotype x environment interaction, metanalysis, informative prior.

\section{Introdução}

O feijão-caupi [Vigna unguiculata (L.) Walp.] é uma das fontes alimentares mais importantes para regiões tropicais e subtropicais do planeta. Atualmente, o Brasil é o terceiro maior produtor mundial da cultura, que é cultivada, sobretudo, nas regiões Norte (55,8 mil hectares) e Nordeste (1,2 milhão de hectare) do País, onde constitui o prato básico das classes de menor poder aquisitivo (Santos et al., 2014).

O feijão-caupi tem sido cultivado em três regiões distintas do Brasil: Centro-oeste, Norte e Nordeste 
(Torres et al., 2015). Isso acarreta dificuldades na recomendação de cultivares, uma vez que as adaptadas a uma determinada condição de cultivo podem não apresentar bom desempenho em outra condição ambiental. Essa resposta diferencial dos genótipos aos diferentes ambientes de cultivo é comumente avaliada por meio da interação genótipo $\mathrm{x}$ ambiente $(\mathrm{GxE})$.

Nesse sentido, alguns trabalhos têm sido conduzidos com o objetivo de selecionar genótipos de feijão-caupi superiores quanto à adaptabilidade e à estabilidade produtiva. As metodologias mais comumente utilizadas para esse fim são as de modelos mistos (REML/BLUP); a de Eberhart \& Russel (1966), baseada na regressão linear simples; e as de Yates \& Cochran (1938) (tradicional) e de Wricke (1965), ambas baseadas na análise de variância (Freire Filho et al., 2002; Rocha et al., 2007; Barros et al., 2013; Torres et al., 2015).

Apesar da grande quantidade de metodologias disponíveis para estudo da interação $\mathrm{GxE}$, poucas fazem uso de informações a priori na estimação dos parâmetros. Nascimento et al. (2011) formularam uma abordagem bayesiana para o método de Eberhart \& Russel (1966), que permitiu maior acurácia na seleção de genótipos de alface, em comparação ao método tradicional. Esta abordagem utiliza três conceitos básicos: uma informação inicial (probabilidade a priori), geralmente assumida como uma lei de probabilidade conjunta sobre os parâmetros, antes de se obter a informação particular das amostras $\mathrm{y}_{1}, \ldots, \mathrm{y}_{\mathrm{n}}$ da variável aleatória; o modelo probabilístico da variável aleatória resposta y, com o qual se obtém a verossimilhança da amostra; e o teorema de Bayes, que combina a informação a priori e a função de verossimilhança, e gera uma distribuição a posteriori (Cotes et al., 2006). Assim, no contexto bayesiano, os parâmetros passam a ser interpretados como variáveis aleatórias, com uma lei de probabilidade (distribuição a priori) que reflete uma informação inicial (ou a falta de informação) sobre eles, independentemente do que os dados possam mostrar (Ramos Molina et al., 2012). Outra peculiaridade dessa análise é que a homogeneidade da variância e a adequação dos resíduos à distribuição normal, pressupostos para muitas metodologias, não são requeridos na abordagem bayesiana (Cotes et al., 2006). Embora a inferência bayesiana seja um procedimento estatístico robusto e tenha muitas aplicações possíveis, sua utilização no melhoramento genético de plantas ainda é pequena.
O objetivo deste trabalho foi selecionar, sob a perspectiva bayesiana, genótipos de feijão-caupi (Vigna unguiculata) que reúnam alta adaptabilidade e estabilidade fenotípicas, no Estado do Mato Grosso do Sul.

\section{Material e Métodos}

Foram conduzidos quatro ensaios de valor de cultivo e uso com 18 linhagens e duas cultivares de feijão-caupi, em 2005 e 2006, nos municípios de Aquidauana, Chapadão do Sul e Dourados (Tabela 1). Utilizou-se o delineamento experimental de blocos ao acaso, com 20 tratamentos e quatro repetições. A unidade experimental foi constituída por quatro fileiras de 5,0 $\mathrm{m}$ de comprimento, espaçadas em $0,5 \mathrm{~m}$ entre fileiras e $0,25 \mathrm{~m}$ entre plantas dentro da fileira. Em cada unidade experimental, a produtividade de grãos foi avaliada nas duas fileiras centrais, tendo-se corrigido a umidade dos grãos para $13 \%$, e extrapolada para $\mathrm{kg} \mathrm{ha}^{-1}$.

As 18 linhagens de feijão-caupi avaliadas foram: MNC99-505G-11, MNC99-507G-4, MNC99-507G-8, MNC99-510G-8, MNC99-510F-16, TE96-290-12G, TE97-309G-18, TE97-304G-4, TE97-304G-12, TE97-309G-24, MNC99-508G-1, MNC99-541F-15, MNC99-541F-18, MNC99-541F-21, MNC99-542F-5, MNC99-542F-7, MNC99-547F-2 e CNC x 409-11F-P2. Além das linhagens, foram avaliadas as cultivares BRS Paraguaçu e BR 17-Gurguéia. Tanto as linhagens quanto as cultivares são oriundas do programa de melhoramento genético de feijão-caupi da Embrapa Meio-Norte.

Para verificar o efeito da interação GxE, o fator de Bayes foi calculado para os dados de produtividade de grãos ajustados a diferentes modelos. Essa abordagem foi utilizada com o intuito de manter toda a apresentação no contexto bayesiano, visto que o fator de Bayes é consistente com a análise de variância clássica (Rouder et al., 2012). Após passarem pela abordagem bayesiana, os dados foram submetidos às análises de adaptabilidade e estabilidade, por meio da metodologia de Eberhart \& Russell (1966).

O modelo de regressão linear de Eberhart \& Russell (1966) adotado foi $Y_{\mathrm{ij}}=\beta_{0 \mathrm{i}}+\beta_{\mathrm{li}} \mathrm{I}_{\mathrm{j}}+\Psi_{\mathrm{ij}}$, em que $\mathrm{Y}_{\mathrm{ij}}$ é a média observada do genótipo i no ambiente $\mathrm{j} ; \beta_{0 \mathrm{i}}$ é o coeficiente linear referente ao i-ésimo genótipo; $\beta_{\mathrm{li}}$ é o coeficiente de regressão do genótipo $\mathrm{i}$; $\mathrm{I}_{\mathrm{j}}$ é o índice 
ambiental j; e $\Psi_{\mathrm{ij}}$ são os erros aleatórios, compostos pelo desvio da regressão do genótipo i no ambiente j e pelo erro médio associado à média. $\mathrm{O}$ índice ambiental foi estimado de acordo com a equação $I_{j}=\bar{Y}-\bar{Y}_{m}$, com

$$
\sum_{j=1}^{n} \mathrm{I}_{\mathrm{j}}=0
$$

em que $\bar{Y}_{m}$ é a média geral; $\bar{Y}_{j}$ é a média do ambiente $\mathrm{j}$; e n é o número de ambientes.

De acordo com o método de Eberhart \& Russell (1966), a adaptabilidade do genótipo foi medida pelo parâmetro $\beta_{1 \mathrm{i}}$, enquantoa estabilidade decomportamento foi avaliada por meio da variância dos desvios da regressão $\left(\sigma_{\text {di }}^{2}\right)$ e pelo coeficiente de determinação $\left(\mathrm{R}^{2}\right)$, que, segundo Cruz \& Regazzi (2007), é uma medida auxiliar de avaliação da estabilidade. Quando a $\sigma_{\text {di }}^{2}$ é significativa e o $\mathrm{R}^{2}$ é superior a $80 \%$, considerase que o $\mathrm{R}^{2}$ indica previsibilidade aceitável. Nessa abordagem frequentista, as hipóteses de interesse, ou seja, $\mathrm{H}_{0}: \beta_{\mathrm{li}}=1$ versus $\mathrm{H}_{1}: \beta_{\mathrm{li}} \neq 1$ e $\mathrm{H}_{0}: \sigma_{\mathrm{di}}^{2}=0$ versus $\mathrm{H}_{1}: \sigma_{\mathrm{di}}^{2}>0$, são avaliadas pelo testes $\mathrm{t}$ e $\mathrm{F}$, respectivamente.

Para a análise bayesiana, foram considerados apenas os genótipos avaliados em ao menos um dos seguintes trabalhos: Freire Filho et al. (2002), Carvalho et al. (2006) e Valadares et al. (2010), utilizados como referência para a especificação das distribuições a priori sob um contexto de metanálise (Tabela 2).

$\mathrm{Na}$ abordagem bayesiana, ao se considerar o modelo estatístico $\mathrm{Y}_{\mathrm{ij}}=\beta_{0 \mathrm{i}}+\beta_{\mathrm{li}} \mathrm{I}_{\mathrm{j}}+\Psi_{\mathrm{ij}}$ e se assumir que cada observação $Y_{\mathrm{ij}}$ tem distribuição $\mathrm{Y}_{\mathrm{ij}} \sim \mathrm{N}\left(\beta_{0 \mathrm{i}}+\beta_{\mathrm{li}} \mathrm{I}_{\mathrm{j}}: \sigma_{\mathrm{di}}^{2}\right)$, a função de verossimilhança para cada genótipo i é dada por:

$$
\begin{aligned}
& \mathrm{L}_{\mathrm{i}}\left(\beta_{0 \mathrm{i}}, \beta_{1 \mathrm{i}}, \sigma_{\mathrm{i}}^{2}, \mathrm{y}_{\mathrm{ij}}\right)=\prod_{\mathrm{j}-1}^{\mathrm{a}-1} \frac{1}{\sqrt{2 \pi \pi_{\mathrm{i}}^{2}}} \exp \left\{-\frac{1}{2 \sigma_{\mathrm{i}}^{2}}\left[\mathrm{y}_{\mathrm{ij}}-\left(\beta_{0 \mathrm{i}}+\beta_{1 \mathrm{i}} \mathrm{I}_{\mathrm{j}}\right)^{2}\right]\right\} \\
& =\frac{1}{\left(\sqrt{2 \pi \sigma_{\mathrm{i}}^{2}}\right)^{2}} \exp \left\{-\frac{1}{2 \sigma_{\mathrm{i}}^{2}} \sum_{\mathrm{j}=1}^{\mathrm{a}}\left[\mathrm{y}_{\mathrm{ij}}-\left(\beta_{0 \mathrm{i}}+\beta_{1 \mathrm{i}} \mathrm{I}_{\mathrm{j}}\right)^{2}\right]\right\}, \forall_{\mathrm{i}} .
\end{aligned}
$$

Para a estimação dos parâmetros de adaptabilidade e estabilidade, é necessário atribuir distribuições a priori para os parâmetros. Para $\beta_{0 \mathrm{i}}+\beta_{\mathrm{li}}$ e $\sigma_{\mathrm{di}}^{2}$, foram consideradas as seguintes distribuições: $\beta_{0 \mathrm{i}} \sim \mathrm{N}\left(\mu_{0 \mathrm{i}}, \sigma_{0 \mathrm{i}}^{2}\right), \beta_{1 \mathrm{i}} \sim \mathrm{N}\left(\mu_{0 \mathrm{i}}, \sigma_{1 \mathrm{i}}^{2}\right)$ e $\sigma_{\mathrm{di}}^{2} \sim \operatorname{GamaInv}\left(\alpha: \beta_{\mathrm{i}}\right) ; \quad$ esta última, uma gama inversa com média e variância iguais a $\beta_{\mathrm{i}} / \alpha_{\mathrm{i}}-1$ e $\beta_{\mathrm{i}}^{2} /\left(\alpha_{\mathrm{i}}-1\right)^{2}\left(\alpha_{\mathrm{i}}-2\right)$, respectivamente.

Ao se assumir a independência entre os parâmetros dessas distribuições, a distribuição a priori conjunta para cada genótipo é dada por:

$$
\begin{aligned}
& \operatorname{Pi}\left(\beta_{0 \mathrm{i}}, \beta_{1 \mathrm{i}}, \sigma_{\mathrm{i}}^{2}, \mathrm{y}_{\mathrm{ij}}\right)=\frac{1}{\sqrt{2 \pi \sigma_{0 \mathrm{i}}^{2}}} \exp \left\{-\frac{1}{2 \sigma_{0 \mathrm{i}}^{2}}\left[\left(\beta_{0 \mathrm{i}}+\mu_{0 \mathrm{i}}\right)^{2}\right]\right\} \times \\
& \frac{1}{\sqrt{2 \pi \sigma_{1 \mathrm{i}}^{2}}} \exp \left\{-\frac{1}{2 \sigma_{\mathrm{li}}^{2}}\left[\left(\beta_{1 \mathrm{i}}+\mu_{1 \mathrm{i}}\right)^{2}\right]\right\} \times \sigma_{0 \mathrm{i}}^{2} \frac{1}{\left[\beta_{\mathrm{i}}^{\alpha \mathrm{i}} \mathrm{G}\left(\alpha_{\mathrm{i}}\right)\right]}\left(\frac{1}{\sigma_{\mathrm{i}}^{2}}\right)^{\alpha_{\mathrm{i}}+1} \\
& \exp \left\{-\frac{1}{\beta_{\mathrm{i}} \sigma_{\mathrm{i}}^{2}}\right\} \infty \exp \left[-\frac{1}{\sigma_{\mathrm{i}}^{2}}\left(\beta_{0 \mathrm{i}}+\mu_{0 \mathrm{i}}\right)^{2}\right] \times \frac{1}{\sqrt{2 \pi \sigma_{1 \mathrm{i}}^{2}}} \\
& \exp \left[-\frac{1}{2 \sigma_{1 \mathrm{i}}^{2}}\left(\beta_{1 \mathrm{i}}+\mu_{1 \mathrm{i}}\right)^{2}\right] \times\left(\frac{1}{\sigma_{\mathrm{i}}^{2}}\right)^{\alpha_{\mathrm{i}}+1} \exp \left\{-\frac{1}{\beta_{\mathrm{i}} \sigma_{\mathrm{i}}^{2}}\right\} .
\end{aligned}
$$

Para fazer inferências sobre os parâmetros de interesse, énecessário obter suas distribuições marginais a posteriori. Ao denotar o vetor de parâmetros para cada genótipo i por $\theta_{\mathrm{pi}}=\left(\beta_{1 \mathrm{i}}, \beta_{2 \mathrm{i}}, \sigma_{3 \mathrm{i}}^{2}\right)$, em que $\mathrm{p}=1,2,3$, a distribuição marginal a posteriori para o parâmetro $\theta_{\mathrm{pi}}$ é obtida pela seguinte integral: $\mathrm{P}\left(\theta_{\mathrm{pi}} \mid \mathrm{x}\right)=\int \mathrm{P}\left(\theta_{\mathrm{pi}} \mid \mathrm{x}\right) \mathrm{d} \theta_{\mathrm{pi}}$, isto é, a integral em relação a todos os parâmetros do vetor, exceto o p-ésimo componente.

Na maioria dos casos, essas integrais são complexas se não apresentam soluções exatas. Para contornar esse problema, utiliza-se outra metodologia: uma amostra da distribuição conjunta a posteriori é obtida ao se utilizar as cadeias de Markov e o método de Monte Carlo para a determinação dos momentos de interesse das distribuições marginais (Cassella \& George, 1992). No presente trabalho, a metodologia foi implementada no programa $\mathrm{R}$ (The R Foundation, 2010), e a amostra

\begin{tabular}{|c|c|c|c|c|c|c|c|}
\hline Ambiente & Ano & Local & Latitude & Longitude & Altitude & Precipitação $^{(1)}(\mathrm{mm})$ & Temperatura média $\left({ }^{\circ} \mathrm{C}\right)$ \\
\hline 1 & 2005 & Aquidauana & $22^{\circ} 01^{\prime} \mathrm{S}$ & $54^{\circ} 05^{\prime} \mathrm{W}$ & $430 \mathrm{~m}$ & 83 & 24,5 \\
\hline 2 & 2005 & Chapadão do Sul & $18^{\circ} 05^{\prime} \mathrm{S}$ & $52^{\circ} 04^{\prime} \mathrm{W}$ & $790 \mathrm{~m}$ & 116 & 22,7 \\
\hline 3 & 2006 & Aquidauana & $22^{\circ} 01^{\prime} \mathrm{S}$ & $54^{\circ} 05^{\prime} \mathrm{W}$ & $430 \mathrm{~m}$ & 121 & 23,2 \\
\hline 4 & 2006 & Dourados & $20^{\circ} 03^{\prime} \mathrm{S}$ & $55^{\circ} 05^{\prime} \mathrm{W}$ & $147 \mathrm{~m}$ & 196 & 21,3 \\
\hline
\end{tabular}
da distribuição conjunta foi obtida por meio da função MCMCregress do pacote MCMCpack. Para avaliar a influência de informações a priori na estimação dos parâmetros de adaptabilidade e estabilidade, optou-se

Tabela 1. Coordenadas geográficas, altitude, precipitação pluvial e temperatura do ar nos quatro ambientes avaliados no Estado do Mato Grosso do Sul.

${ }^{(1)}$ Precipitação acumulada ao longo do experimento. 
pela utilização de dois modelos diferentes (Nascimento et al., 2011).

No primeiro modelo (M1), foram consideradas distribuições a priori informativas, cujas informações eram provenientes da aplicação da técnica de metanálise, que se caracteriza pela utilização de informações dos trabalhos anteriores de Freire Filho et al. (2002), Carvalho et al. (2006) e Valadares et al. (2010). Dessa forma, todos os genótipos avaliados nos experimentos apresentados foram considerados para a análise bayesiana (Tabela 2). As informações foram inseridas na análise por meio dos valores assumidos para os parâmetros das distribuições a priori, denominados de hiperparâmetros.

Esses valores foram dados pela média e pela variância da amostra composta pelas estimativas dos parâmetros obtidas das referências citadas (Tabela 2), o que resultou nas distribuições:

$\beta_{0 \mathrm{i}} \sim \mathrm{N}\left(\mu_{0 \mathrm{i}}=\bar{\beta}_{0 \mathrm{i}} \sigma_{1 \mathrm{i}}^{2}=\operatorname{Var}\left(\bar{\beta}_{0 \mathrm{i}}\right)\right), \quad \beta_{1 \mathrm{i}} \sim \mathrm{N}\left(\mu_{1 \mathrm{i}}=\bar{\beta}_{1 \mathrm{i}}, \sigma_{1 \mathrm{i}}^{2}=\operatorname{Var}\left(\bar{\beta}_{0 \mathrm{i}}\right)\right)$, e $\sigma_{\mathrm{di}}^{2} \sim \operatorname{GamaInv}\left(\alpha_{\mathrm{i}}, \beta_{\mathrm{i}}\right)$, em que $\bar{\beta}_{0 \mathrm{i}}$, são as médias das estimativas dos $\beta_{0 i} ; \bar{\beta}_{1 \mathrm{i}}$, são as médias das estimativas $\operatorname{dos} \bar{\beta}_{1 \mathrm{i}} ; \operatorname{Var}\left(\bar{\beta}_{0 \mathrm{i}}\right)$ é a variância dos valores médios de $\bar{\beta}_{0 \mathrm{i}} ; \operatorname{Var}\left(\bar{\beta}_{1 \mathrm{i}}\right)$ é variância dos valores médios de $\bar{\beta}_{1 \mathrm{i}}$; e $\alpha_{\mathrm{i}}$ e $\beta_{\mathrm{i}}$ são os valores obtidos pela resolução do sistema:

Tabela 2. Estimativas da média $\left(\beta_{0 \mathrm{i}}\right)$ e dos parâmetros de adaptabilidade $\left(\beta_{\mathrm{li}}\right)$ e estabilidade $\left(\sigma_{\mathrm{di}}^{2}\right)$ obtidas com a metodologia de Eberhart \& Russel (1966), nos trabalhos de Freire Filho et al. (2002), Carvalho et al. (2006) e Valadares et al. (2010).

\begin{tabular}{llccc}
\hline Genótipo & Referência & $\beta_{0 \mathrm{i}}$ & $\beta_{1 \mathrm{i}}$ & $\sigma_{\mathrm{di}}^{2}$ \\
\hline MNC99-505G-11 & Carvalho et al. (2006) & 1.129 & 0,910 & $179.451,5$ \\
MNC99-507G-4 & Carvalho et al. (2006) & 1.293 & 0,920 & $155.007,7$ \\
MNC99-507G-8 & Carvalho et al. (2006) & 1.214 & 1,010 & $244.123,9$ \\
TE96-290-12G & Carvalho et al. (2006) & 986 & 0,820 & $164.779,2$ \\
MNC99-510G-8 & Carvalho et al. (2006) & 1.216 & 1,180 & $182.460,2$ \\
MNC99-510F-16 & Carvalho et al. (2006) & 1.205 & 1,290 & $160.836,2$ \\
TE97-309G-18 & Carvalho et al. (2006) & 1.075 & 1,000 & $262.266,4$ \\
TE97-304G-4 & Carvalho et al. (2006) & 953 & 0,720 & $236.565,9$ \\
TE97-304G-12 & Carvalho et al. (2006) & 1.286 & 1,200 & $190.453,6$ \\
TE97-309G-24 & Carvalho et al. (2006) & 1.147 & 0,960 & $370.671,6$ \\
MNC99-508G-1 & Carvalho et al. (2006) & 1.101 & 0,720 & $94.737,2$ \\
MNC99-541F-15 & Carvalho et al. (2006) & 933 & 0,880 & $16.558,3$ \\
MNC99-541F-18 & Carvalho et al. (2006) & 1.112 & 1,050 & $271.804,5$ \\
MNC99-541F-21 & Carvalho et al. (2006) & 1.028 & 0,850 & $108.127,3$ \\
MNC99-542F-5 & Valadares et al. (2010) & 1.229 & 0,932 & $78.945,8$ \\
MNC99-542F-7 & Carvalho et al. (2006) & 981 & 0,990 & $77.878,3$ \\
MNC99-547F-2 & Carvalho et al. (2006) & 965 & 0,770 & $58.694,5$ \\
BRS Paraguaçu & Carvalho et al. (2006) & 1.064 & 1,340 & $66.037,9$ \\
BR 17-Gurguéia & Freire Filho et al. (2002) & 881 & 1,280 & 952.000 \\
CNC x 409-11F-P2 & Carvalho et al. (2006) & 1.079 & 1,040 & $79.216,7$ \\
\hline
\end{tabular}

$$
\left\{\begin{array}{l}
\bar{\sigma}_{\mathrm{i}}^{2}=\beta_{\mathrm{i}} / \alpha_{\mathrm{i}}-1 \\
\operatorname{Var}\left(\bar{\sigma}_{\mathrm{i}}^{2}\right)=\beta_{\mathrm{i}}^{2} /\left(\alpha_{\mathrm{i}}-1\right)^{2}\left(\alpha_{\mathrm{i}}-2\right), \text { que foi: } \\
\alpha_{\mathrm{i}}=\left(\sigma_{\mathrm{i}}^{2}\right)^{3} / \operatorname{Var}\left(\bar{\sigma}_{\mathrm{i}}^{2}\right) \times \bar{\sigma}_{\mathrm{i}}^{2} \quad \beta_{\mathrm{i}}=\left(\sigma_{\mathrm{i}}^{2}\right)^{3} / \operatorname{Var}\left(\bar{\sigma}_{\mathrm{i}}^{2}\right)+\bar{\sigma}_{\mathrm{i}}^{2}
\end{array}\right.
$$

No segundo modelo (M2), foram utilizadas distribuições a priori pouco informativas, que representam distribuições de probabilidade com grande variância. Foram adotadas as seguintes distribuições: $\beta_{0 \mathrm{i}} \sim \mathrm{N}\left(\mu_{0 \mathrm{i}}=\bar{\beta}_{0 \mathrm{i}} \sigma_{0 \mathrm{i}}^{2}=1.000 .000\right), \beta_{\mathrm{li}} \sim \mathrm{N}\left(\mu_{0 \mathrm{i}}=\bar{\beta}_{0 \mathrm{i}} \sigma_{1 \mathrm{i}}^{2}=100.000\right)$ e $\sigma_{\mathrm{i}}^{2}$ $\sim \operatorname{GamaInv}\left(\alpha_{\mathrm{i}}=0,00001 ; \quad \beta_{\mathrm{i}}=5.000\right) . \quad$ A Figura 1
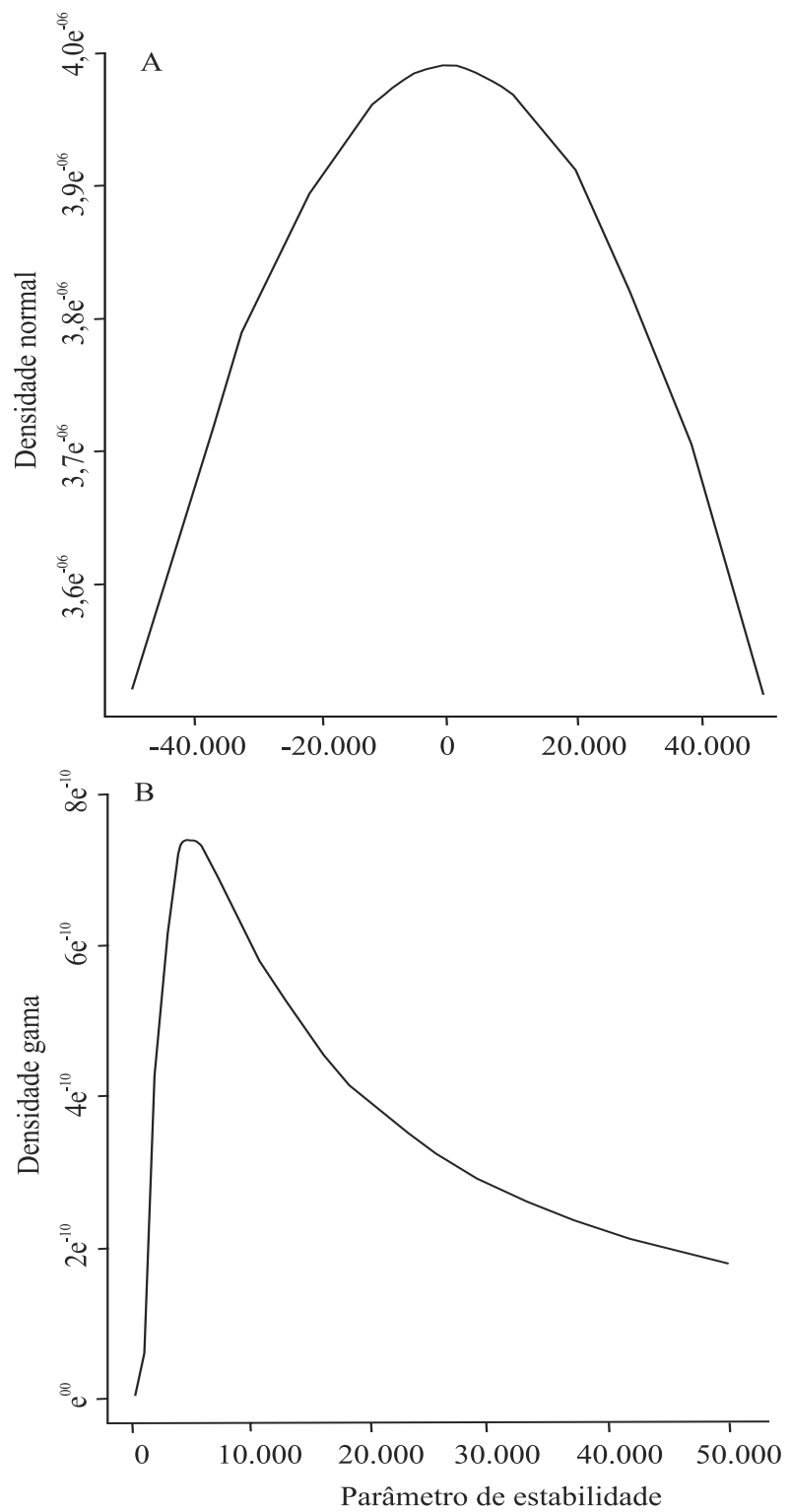

Figura 1. Distribuições pouco informativas utilizadas como informação a priori: A, normal $(\mu=0$ e $\left.\sigma_{\mathrm{d}}^{2}=100.000\right) ;$ e $\mathrm{B}$, gama inversa $(\mathrm{a}=0,00001$ $\mathrm{e} \mathrm{b}=5.000)$. 
apresenta as distribuições pouco informativas obtidas por meio dos hiperparâmetros fornecidos, de acordo com Nascimento et al. (2011).

A comparação entre M1 e M2, ou seja, entre as distribuições a priori informativas e não informativas, foi feita pelo fator de Bayes (Kass \& Raftery, 1995). O cálculo do fator de Bayes foi realizado por meio da função Bayes Factor do pacote MCMCpack. Conforme Jeffreys (1961), o fator de Bayes pode ser interpretado da seguinte forma: FBij $<1$ mostra evidência a favor do modelo j; $1 \leq \mathrm{FBij}<3$ mostra evidência moderada a favor do modelo i; $3 \leq \mathrm{FBij}<10$ mostra evidência substancial a favor do modelo $\mathrm{i} ; 10 \leq \mathrm{FBij}<30$ mostra evidência forte a favor do modelo i; $30 \leq \mathrm{FBij}<100$ mostra evidência muito forte a favor do modelo $\mathrm{i}$; e FBij $\geq 100$ mostra evidência decisiva a favor do modelo i.

Com relação ao parâmetro de estabilidade $\left(\sigma_{\mathrm{di}}^{2}\right)$, as amostras de sua distribuição marginal foram obtidas indiretamente, uma vez que esse parâmetro representa uma função de $\sigma_{i}^{2}$. Ao se obter valores para $\sigma_{i}^{2}$ indiretamente, em cada iteração, obtêm-se valores para $\sigma_{\mathrm{di}}^{2}$, por meio da seguinte expressão: $\widehat{\sigma}_{\mathrm{di}}^{2}=\widehat{\sigma}_{\mathrm{i}}^{2}-(\mathrm{QMR} / \mathrm{r})$, em que QMR é o quadrado médio do resíduo fornecido pela análise da variância; e $r$ é número de repetições do experimento.

As hipóteses de interesse foram testadas pela construção de intervalos de credibilidade para os parâmetros, obtidos diretamente da distribuição marginal a posteriori dos parâmetros. Assim, seja $\theta_{\mathrm{i}}=\left(\beta_{0 \mathrm{i}}: \beta_{1 \mathrm{i}}: \sigma_{\mathrm{di}}^{2}\right)$ o vetor de parâmetros, o intervalo de credibilidade para $\theta_{\mathrm{i}}$, com probabilidade de cobertura $\delta$, foi dado por:

$$
\begin{aligned}
& \int_{-\infty}^{\theta^{*}} \mathrm{P}_{\mathrm{i}}\left(\theta_{\mathrm{i}}=\left(\beta_{0 \mathrm{i}}: \beta_{1 \mathrm{i}}: \sigma_{\mathrm{di}}^{2}\right) \mid \mathrm{y}_{\mathrm{ij}}\right) \mathrm{d} \theta_{\mathrm{i}}=\alpha / 2 \mathrm{e} \\
& \int_{\theta^{*}}^{-\infty} \mathrm{P}_{\mathrm{i}}\left(\theta_{\mathrm{i}}=\left(\beta_{0 \mathrm{i}}: \beta_{1 \mathrm{i}}: \sigma_{\mathrm{di}}^{2}\right) \mid \mathrm{y}_{\mathrm{ij}}\right) \mathrm{d} \theta_{\mathrm{i}}=\alpha / 2,
\end{aligned}
$$

em que $\theta_{*}$ e $\theta^{*}$ representam, respectivamente, os limites inferiores e superiores do intervalo de credibilidade.

Como o Gibbs sampler é um algoritmo iterativo, foi necessário verificar sua convergência, que, no presente trabalho, foi constatada pela aplicação dos critérios de Heidelberger \& Welch (1983), Geweke (1992) e Raftery \& Lewis (1992), implementados no pacote Bayesian Output Analysis (BOA) do programa R (The R Foundation, 2010).

$\mathrm{Na}$ análise bayesiana de adaptabilidade e estabilidade, foram consideradas 110.000 iterações no algoritmo Gibbs sampler para cada parâmetro do modelo de regressão adotado, com um período de aquecimento ("burn-in") de 10.000 iterações. Para obter-se uma amostra não correlacionada, considerouse um espaçamento entre os pontos amostrados de duas iterações ("thinning"), o que resultou em uma amostra final de tamanho 50.000. As amostras representam amostras das distribuições marginais a posteriori de cada parâmetro, sob as quais foi feita a inferência de cada parâmetro.

\section{Resultados e Discussão}

O fator de Bayes obtido da comparação entre o modelo completo [M1 (numerador): bloco/ambiente + genótipos + ambientes + GxA] e o modelo restrito [M2 (denominador): bloco/ambiente + genótipos + ambientes] foi de 8.630. Esse resultado é indicativo de ocorrência de resposta diferenciada dos genótipos de acordo com o ambiente, e está de acordo com as diferenças observadas entre os ambientes em termos de altitude, latitude, longitude, precipitação e temperatura (Tabela 1). Efeitos significativos de genótipos, ambientes e da interação $\mathrm{GxE}$ foram relatados em diversos trabalhos com genótipos de feijão-caupi de porte semiprostrado avaliados em ensaios multiambientes, na região Meio-Norte do Brasil (Freire Filho et al., 2002; Rocha et al., 2007; Barros et al., 2013; Torres et al., 2015).

Em relação à convergência entre as cadeias simuladas, o fator de dependência de Raftery \& Lewis (1992) forneceu valores menores que cinco, e o valor $p$ do critério de Geweke (1992) foi sempre maior que o nível de significância pré-fixado (5\%). Portanto, ambos os critérios indicaram a convergência das cadeias geradas pelo algoritmo Gibbs sampler, confirmada pelo critério de Heidelberger \& Welch (1983), que verifica se os valores das cadeias são provenientes de distribuição estacionária.

As estimativas dos parâmetros de adaptabilidade e estabilidade foram obtidas pelo cálculo da média a posteriori (Tabela 3). No M1 (prioris informativas), dos 20 genótipos avaliados com a metodologia bayesiana, apenas os genótipos MNC99-507G-4, TE97-309G-24, MNC99-542F-7 e BR 17-Gurguéia foram classificados como de adaptabilidade específica a ambientes favoráveis $\left(\mathrm{LI} \bar{\beta}_{1 \mathrm{i}}>1\right)$. Os genótipos TE96-290-12G, MNC99-510F-16, MNC99-508G-1, MNC99-541F-21, MNC99-542F-5 e MNC99-547F-2 
apresentaram adaptabilidade específica a ambientes desfavoráveis $\left(\operatorname{LS} \bar{\beta}_{1 \mathrm{i}}<1\right)$. Os demais genótipos foram classificados como de adaptabilidade e estabilidade geral, com valores dos parâmetros de adaptabilidade e estabilidade dentro dos índices de confiabilidade de
$95 \%$. Entretanto, com a análise dos genótipos por meio do M2 (prioris não informativas), observou-se que nenhum genótipo apresentou adaptabilidade específica a ambientes favoráveis $\left(\operatorname{LI} \bar{\beta}_{1 \mathrm{i}}>1\right)$. Desse modo, é possível inferir que a utilização do modelo frequentista,

Tabela 3. Estimativas da média a posteriori $\left(\bar{\beta}_{0 \mathrm{i}}\right)$ e dos intervalos de credibilidade (95\%) dos parâmetros de adaptabilidade $\left(\bar{\beta}_{1 \mathrm{i}}\right)$ e estabilidade $\left(\bar{\sigma}_{\mathrm{di}}^{2}\right)$, ao se considerar prioris informativas e não informativas para os genótipos de feijão-caupi (Vigna unguiculata) semiprostrado ${ }^{(1)}$.

\begin{tabular}{|c|c|c|c|c|c|c|c|c|c|c|}
\hline Genótipo & $\mathrm{LI} \bar{\beta}_{0 \mathrm{i}}$ & $\bar{\beta}_{0 \mathrm{i}}$ & $\operatorname{LS} \bar{\beta}_{0 \mathrm{i}}$ & LI $\bar{\beta}_{1 \mathrm{i}}$ & $\bar{\beta}_{1 \mathrm{i}}$ & $\operatorname{LS} \bar{\beta}_{1 \mathrm{i}}$ & $\bar{\sigma}_{i}^{2}$ & $\mathrm{LI} \bar{\sigma}_{\mathrm{di}}^{2}$ & $\bar{\sigma}_{\mathrm{di}}^{2}$ & $\mathrm{LS} \bar{\sigma}_{\mathrm{di}}^{2}$ \\
\hline & \multicolumn{10}{|c|}{ Prioris informativas } \\
\hline MNC99-505G-11 & 465,32 & 486,90 & 510,74 & 0.88 & 0,95 & 1,02 & 513,98 & $-5.371,38$ & $-5.107,38$ & $-4.577,60$ \\
\hline MNC99-507G-4 & 497,67 & 517,76 & 540,25 & 1,06 & 1,12 & 1,18 & 446,40 & $-5.405,53$ & $-5.174,96$ & $-4.708,37$ \\
\hline MNC99-507G-8 & 514,93 & 560,65 & 617,32 & 0,74 & 0,87 & 1,01 & $2.306,42$ & $-4.544,90$ & $-3.314,93$ & $-664,95$ \\
\hline TE96-290-12G & 614,02 & 631,36 & 649,52 & 0,65 & 0,70 & 0,75 & 323,78 & $-5.462,76$ & $-5.297,57$ & $-4.972,81$ \\
\hline MNC99-510G-8 & 516,41 & 572,32 & 646,05 & 0,79 & 0,94 & 1,11 & $3.455,58$ & $-4.073,34$ & $-2.165,78$ & $2.173,62$ \\
\hline MNC99-510F-16 & 549,87 & 558,03 & 566,56 & 0,81 & 0,84 & 0,87 & 71,21 & $-5.586,48$ & $-5.550,15$ & $-5.478,34$ \\
\hline TE97-309G-18 & 372,36 & 400,04 & 431,77 & 1,37 & 1,46 & 1,54 & 850,77 & $-5.218,43$ & $-4.770,58$ & $-3.842,97$ \\
\hline TE97-304G-4 & 557,03 & 612,15 & 674,94 & 0,87 & 1,05 & 1,20 & $3.540,21$ & $-3.950,82$ & $-2.081,14$ & $1.776,12$ \\
\hline TE97-304G-12 & 681,57 & 728,14 & 784,57 & 0,76 & 0,89 & 1,04 & $2.435,33$ & $-4.490,23$ & $-3.186,02$ & $-398,04$ \\
\hline TE97-309G-24 & 598,73 & 653,85 & 721,13 & 1,13 & 1,31 & 1,46 & $3.449,07$ & $-4.042,24$ & $-2.172,29$ & $1.899,53$ \\
\hline MNC99-508G-1 & 685,04 & 740,72 & 804,03 & 0,59 & 0,75 & 0,91 & $3.580,37$ & $-3.886,39$ & $-2.040,99$ & $1.696,26$ \\
\hline MNC99-541F-15 & 613,83 & 631,48 & 649,91 & 0,98 & 1,04 & 1,09 & 335,18 & $-5.457,13$ & $-5.286,18$ & $-4.949,75$ \\
\hline MNC99-541F-18 & 648,75 & 696,55 & 751,06 & 0,84 & 0,98 & 1,12 & $2.600,63$ & $-4.364,81$ & $-3.020,73$ & $-278,95$ \\
\hline MNC99-541F-21 & 463,17 & 466,40 & 469,70 & 0,85 & 0,86 & 0,87 & 10,96 & $-5.615,98$ & $-5.610,39$ & $-5.599,43$ \\
\hline MNC99-542F-5 & 787,04 & 802,96 & 819,63 & 0,82 & 0,87 & 0,92 & 272,08 & $-5.488,08$ & $-5.349,28$ & $-5.075,84$ \\
\hline MNC99-542F-7 & 528,43 & 553,57 & 580,64 & 1,27 & 1,35 & 1,43 & 692,81 & $-5.286,39$ & $-4.928,55$ & $-4.214,91$ \\
\hline MNC99-547F-2 & 408,49 & 433,16 & 460,17 & 0,82 & 0,89 & 0,97 & 671,43 & $-5.294,76$ & $-4.949,93$ & $-4.260,79$ \\
\hline BRS Paraguaçu & 752,24 & 832,31 & 922,38 & 0,82 & 1,03 & 1,27 & $8.004,60$ & $-1.880,35$ & $2.383,24$ & $11.157,78$ \\
\hline BR 17-Gurguéia & 689,52 & 727,79 & 767,74 & 1,03 & 1,15 & 1,26 & $1.610,01$ & $-4.829,86$ & $-4.011,35$ & $-2.410,51$ \\
\hline \multirow[t]{2}{*}{ CNC x 409-11F-P2 } & 467,05 & 536,30 & 626,26 & 0,86 & 1,04 & 1,23 & $5.430,32$ & $-3.135,87$ & $-191,03$ & $6.295,22$ \\
\hline & \multicolumn{10}{|c|}{ Prioris não informativas } \\
\hline MNC99-505G-11 & 308,71 & 480,80 & 650,20 & 0,44 & 0,95 & 1,46 & $77.320,00$ & $-3.937,15$ & $71.698,64$ & $239.078,60$ \\
\hline MNC99-507G-4 & 346,56 & 511,36 & 673,70 & 0,64 & 1,13 & 1,61 & $71.428,68$ & $-4.076,13$ & $65.807,32$ & $218.978,60$ \\
\hline MNC99-507G-8 & 242,50 & 533,50 & 819,00 & $-0,01$ & 0,85 & 1,71 & $207.000,00$ & $-7.65,36$ & $201.378,60$ & $678.978,60$ \\
\hline TE96-290-12G & 475,96 & 628,80 & 779,10 & 0,24 & 0,70 & 1,15 & $62.010,00$ & $-4.298,38$ & $56.388,64$ & $187.178,60$ \\
\hline MNC99-510G-8 & 196,20 & 532,80 & 862,57 & $-0,11$ & 0,89 & 1,88 & $272.500,00$ & 909,39 & $266.878,60$ & $910.111,60$ \\
\hline MNC99-510F-16 & 436,11 & 556,96 & 675,90 & 0,48 & 0,84 & 1,19 & $39.859,66$ & $-4.796,71$ & $34.238,30$ & $114.978,60$ \\
\hline TE97-309G-18 & 190,75 & 389,70 & 586,20 & 0,89 & 1,49 & 2,07 & $101.800,00$ & $-3.352,77$ & $96.178,64$ & $323.478,60$ \\
\hline TE97-304G-4 & 236,60 & 589,30 & 934,10 & 0,08 & 1,12 & 2,17 & $297.600,00$ & $1.549,64$ & $291.978,60$ & $997.378,60$ \\
\hline TE97-304G-12 & 404,63 & 703,30 & 993,40 & $-0,04$ & 0,84 & 1,72 & $216.000,00$ & $-567,89$ & $210.378,60$ & $710.378,60$ \\
\hline TE97-309G-24 & 280,83 & 622,70 & 956,00 & 0,38 & 1,39 & 2,39 & $279.500,00$ & $1.072,16$ & $273.878,60$ & $931.778,60$ \\
\hline MNC99-508G-1 & 351,69 & 715,80 & $1.069,00$ & $-0,31$ & 0,76 & 1,83 & $314.800,00$ & $1.965,77$ & $309.178,60$ & $1.051 .379,60$ \\
\hline MNC99-541F-15 & 474,99 & 629,18 & 780,70 & 0,59 & 1,04 & 1,50 & $63.005,38$ & $-4.275,55$ & $57.384,02$ & $190.378,60$ \\
\hline MNC99-541F-18 & 360,50 & 675,90 & 983,40 & 0,04 & 0,96 & 1,89 & $240.300,00$ & 52,64 & $234.678,60$ & $793.278,60$ \\
\hline MNC99-541F-21 & 354,47 & 466,10 & 576,40 & 0,53 & 0,86 & 1,19 & $34.430,00$ & $-4.915,28$ & $28.808,64$ & $97.678,64$ \\
\hline MNC99-542F-5 & 653,15 & 800,30 & 944,60 & 0,44 & 0,87 & 1,30 & $57.820,00$ & $-4.400,44$ & $52.198,64$ & $172.778,60$ \\
\hline MNC99-542F-7 & 359,27 & 547,80 & 733,50 & 0,81 & 1,37 & 1,92 & $91.937,00$ & $-3.595,87$ & $86.315,64$ & $286.978,60$ \\
\hline MNC99-547F-2 & 239,48 & 426,50 & 610,80 & 0,34 & 0,90 & 1,45 & $90.290,00$ & $-3.628,51$ & $84.668,64$ & $283.678,60$ \\
\hline BRS Paraguaçu & 287,01 & 795,10 & $1.280,00$ & $-0,61$ & 0,88 & 2,37 & $587.700,00$ & $9.281,97$ & $582.078,60$ & $2.022 .379,60$ \\
\hline BR 17-Gurguéia & 461,08 & 721,81 & 976,30 & 0,36 & 1,13 & 1,90 & $168.691,04$ & $-1.752,15$ & $163.069,70$ & $546.078,60$ \\
\hline $\mathrm{CNC} \times$ 409-11F-P2 & 67,12 & 484,20 & 892,10 & $-0,19$ & 1,04 & 2,28 & $408.700,00$ & $4.519,95$ & $403.078,60$ & $1.392 .379,60$ \\
\hline
\end{tabular}

${ }^{(1)} \mathrm{LI}$, limite inferior; e LS, limite superior. 
em que informações a priori não são levadas em conta, tende a atribuir aos genótipos adaptabilidade e estabilidade a ambientes diversos, o que compromete a recomendação acurada para o cultivo.

Na comparação entre o M1 e o M2, foram observadas diferenças de magnitudes semelhantes entre os parâmetros. Portanto, é necessário que se informe qual dos dois modelos apresenta maior qualidade de ajuste, a partir do cálculo do fator de Bayes (Nascimento et al., 2011). Com exceção do genótipo MNC99-541F-21, todos os demais apresentaram FB $>10$ (Tabela 4), o que indica que o uso de prioris informativas fornece resultados mais acurados. Apesar de a informação a priori para cada genótipo ter sido baseada em apenas um trabalho, resultados similares a esses foram relatados em estudos semelhantes com avaliação da produtividade de matéria seca de genótipos de alfafa (Medicago sativa L.) (Nascimento et al., 2011) e da produtividade de grãos de milho-pipoca (Zea mays L.) (Couto et al., 2015). Assim, espera-se que os resultados sejam ainda mais precisos, em trabalhos com maior disponibilidade de informação a priori, obtida pela metanálise.

Tabela 4. Valores do fator de Bayes obtidos na comparação entre os modelos com prioris informativas (i) e não informativas (j), para os genótipos de feijão-caupi (Vigna unguiculata) semiprostrado.

\begin{tabular}{lc}
\hline Genótipo & $\mathrm{FB}_{\mathrm{ij}}$ \\
\hline MNC99-505G-11 & 31,8 \\
MNC99-507G-4 & 37,7 \\
MNC99-507G-8 & 43,8 \\
TE96-290-12G & 18,7 \\
MNC99-510G-8 & 46,9 \\
MNC99-510F-16 & 19,4 \\
TE97-309G-18 & 40,1 \\
TE97-304G-4 & 37,7 \\
TE97-304G-12 & 41,4 \\
TE97-309G-24 & 42,0 \\
MNC99-508G-1 & 36,7 \\
MNC99-541F-15 & 17,9 \\
MNC99-541F-18 & 35,8 \\
MNC99-541F-21 & 2,2 \\
MNC99-542F-5 & 19,0 \\
MNC99-542F-7 & 28,1 \\
MNC99-547F-2 & 29,6 \\
BRS Paraguaçu & 41,5 \\
BR 17-Gurguéia & 27,4 \\
CNC x 409-11F-P2 & 45,4 \\
\hline
\end{tabular}

\section{Conclusões}

1. A abordagem bayesiana proporciona maior acurácia na seleção de genótipos de feijão-caupi (Vigna unguiculata) semiprostrado com alta adaptabilidade e estabilidade fenotípicas.

2. Com base nas prioris informativas, os genótipos MNC99-507G-4, TE97-309G-24, MNC99-542F-7 e BR 17-Gurguéia são classificados como de adaptabilidade específica a ambientes favoráveis.

3. Os genótipos TE96-290-12G, MNC99-510F-16, MNC99-508G-1, MNC99-541F-21, MNC99-542F-5 e MNC99-547F-2 apresentam adaptabilidade específica a ambientes desfavoráveis.

\section{Agradecimentos}

À Coordenação de Aperfeiçoamento de Pessoal de Nível Superior (Capes) e à Fundação Arthur Bernardes (Funarbe), pela concessão de bolsas.

\section{Referências}

BARROS, M.A.; ROCHA, M. de M.; GOMES, R.L.F.; SILVA, K.J.D. e; NEVES, A.C. das. Adaptabilidade e estabilidade produtiva de feijão-caupi de porte semiprostrado. Pesquisa Agropecuária Brasileira, v.48, p.403-410, 2013. DOI: 10.1590/ S0100-204X2013000400008.

CARVALHO, H.W.L. de; BRITO NETO, J.; FREIRE FILHO, F.R.; ROCHA, M. de M.; RIBEIRO, V.Q.; RODRIGUES, A.R. dos S.; RIBEIRO, S.S.; NOGUEIRA, L.C. Adaptabilidade e estabilidade de linhagens de feijão-caupi de porte prostrado em Sergipe e Alagoas. In: CONGRESSO NACIONAL DE FEIJÃO-CAUPI, 2006, Teresina. Anais eletrônicos... Teresina: Embrapa Meio Norte, 2006. Disponível em: <http://www.cpamn.embrapa.br/ anaisconac2006/listaresumo.htm>. Acesso em: 3 fev. 2015.

CASSELLA, G.; GEORGE, E.I. Explaining the Gibbs sampler. The American Statistician, v.46, p.167-174, 1992.

COTES, J.M.; CROSSA, J.; SANCHES, A.; CORNELIUS, P.A. A Bayesian approach for assessing the stability of genotypes. Crop Science, v.46, p.2654-2665, 2006. DOI: 10.2135/ cropsci2006.04.0227.

COUTO, M.F.; NASCIMENTO, M.; AMARAL, A.T. do; SILVA, F.F. e; VIANA, A.P.; VIVAS, M. Eberhart and Russel's Bayesian method in the selection of popcorn cultivars. Crop Science, v.55, p.571-577, 2015. DOI: 10.2135/cropsci2014.07.0498.

CRUZ, C.D.; REGAZZI, A.J. Modelos biométricos aplicados ao melhoramento genético. Viçosa: Imprensa Universitária, 2007. 480p.

EBERHART, S.A.; RUSSELL, W.A. Stability parameters for comparing varieties. Crop Science, v.6, p.36-40, 1966. DOI: 10.2135/cropsci1966.0011183X000600010011x. 
FREIRE FILHO, F.R.; RIBEIRO, V.Q.; ROCHA, M. de M.; LOPES, Â.C. de A. Adaptabilidade e estabilidade da produtividade de grãos de linhagens de caupi de porte enramador. Revista Ceres, v.49, p.383-393, 2002.

GEWEKE, J. Evaluating the accuracy of sampling-based approaches to the calculation of posterior moments. In: BERNARDO, J.M.; BERGER, J.O.; DAVID, A.P.; SMITH, A.F.M. (Ed.). Bayesian statistics. New York: Oxford University, 1992. p.625-631.

HEIDELBERGER, P.; WELCH, P.D. Simulation run length control in the presence of an initial transient. Operations Research, v.31, p.1109-1144, 1983. DOI: 10.1287/opre.31.6.1109.

JEFFREYS, H. Theory of probability. $3^{\text {rd }}$ ed. Oxford: Claredon, 1961. 447p.

KASS, R.E.; RAFTERY, A.E. Bayes factors. Journal of the American Statistical Association, v.90, p.773-795, 1995. DOI: 10.1080/01621459.1995.10476572.

NASCIMENTO, M.; SILVA, F.F.; SÁFADI, T.; NASCIMENTO, A.C.M.C.; FERREIRA, R. de P.; CRUZ, C.D. Abordagem bayesiana para avaliação da adaptabilidade e estabilidade de genótipos de alfafa. Pesquisa Agropecuária Brasileira, v.46, p.26-32, 2011. DOI: 10.1590/S0100-204X2011000100004.

RAFTERY, A.E.; LEWIS, S.M. Comment: one long run with diagnostics: implementation strategies for Markov chain Monte Carlo. Statistical Science, v.7, p.493-497, 1992. DOI: 10.1214/ ss/1177011143.

RAMOS MOLINA, L.M.; SANCHE, A.; COTES TORRES, J.M. Inferência bayesiana na análise de testes regionais de arroz em dois sistemas de cultivo. Revista Facultad Nacional de Agronomía, v.64, p.5883-5891, 2012.

ROCHA, M. de M.; FREIRE FILHO, F.R.; RIBEIRO, V.Q.; CARVALHO, H.W.L. de; BELARMINO FILHO, J.; RAPOSO, J.A.A.; ALCÂNTARA, J. dos P.; RAMOS, S.R.R.; MACHADO,
C. de F. Adaptabilidade e estabilidade produtiva de genótipos de feijão-caupi de porte semi-ereto na Região Nordeste do Brasil. Pesquisa Agropecuária Brasileira, v.42, p.1283-1289, 2007. DOI: 10.1590/S0100-204X2007000900010.

ROUDER, J.N.; MOREY, R.D.; SPECKMAN, P.L.; PROVINCE, J.M. Default Bayes factors for ANOVA designs. Journal of Mathematical Psychology, v.56, p.356-374, 2012. DOI: 10.1016/j. jmp.2012.08.001.

SANTOS, J.A. da S.; SOARES, C.M.G.; CORRÊA, A.M.; TEODORO, P.E.; RIBEIRO, L.P.; ABREU, H.K.A. de. Agronomic performance and genetic dissimilarity among cowpea (Vigna unguiculata (L.) Walp.) genotypes. Global Advanced Research Journal of Agricultural Science, v.3, p.271-277, 2014.

THE R FOUNDATION. R: the R project for statistical computing. Vienna, 2010. Available at: <https://www.r-project.org > . Accessed on: 15 Apr. 2015.

TORRES, F.E.; SAGRILO, E.; TEODORO, P.E.; RIBEIRO, L.P.; CARGNELUTTI FILHO, A. Número de repetições para avaliação de caracteres em genótipos de feijão-caupi. Bragantia, v.74, p.161-168, 2015. DOI: 10.1590/1678-4499.0393.

VALADARES, R. de N.; MOURA, M. da C.C.L.; SILVA, A.F.A. da; SILVA, L.S. da; VASCONCELOS, M. da C.C. de A.; SILVA, R.G. Adaptabilidade e estabilidade fenotípica em genótipos de feijão-caupi (Vigna unguiculata (L.) Walp.) de porte ereto/ semi-ereto nas mesorregiões leste e sul maranhense. Agropecuária Científica no Semi-árido, v.6, p.21-27, 2010.

WRICKE, G. Zur berechnung der okovalenz bei sommerweizen und hafer. Zeitschrift für Pflanzenzüchtung, v.52, p.127-138, 1965.

YATES, F.; COCHRAN, W.G. The analysis of group of experiments. The Journal of Agricultural Science, v.28, p.556-580, 1938. DOI: $10.1017 /$ S0021859600050978.

$\overline{\text { Recebido em } 13 \text { de abril de } 2015 \text { e aprovado em } 11 \text { de agosto de } 2015}$

Pesq. agropec. bras., Brasília, v.50, n.10, p.878-885, out. 2015 DOI: $10.1590 / \mathrm{S} 0100-204 X 2015001000003$ 